\title{
Resistencia antimicrobiana en Staphylococcus coagulasa positiva (CoPS) aislados de perros con otitis externa
}

\author{
Antimicrobial resistance in coagulase-positive staphylococci (CoPS) isolated \\ from dogs with external otitis
}

Joel André Palomino-Farfán ${ }^{1,2}$, Luis Alvarez V. ${ }^{1}$, Juan Siuce M. ${ }^{1}$, Sonia Calle E. ${ }^{1}$

\section{Resumen}

\begin{abstract}
El grupo de Staphylococcus coagulasa positiva (CoPS) incluye a las especies $S$. aureus, $S$. pseudintermedius, $S$. intermedius y a $S$. schleiferi subsp. coagulans. El propósito de este estudio fue evaluar la resistencia antimicrobiana de CoPS aislados de perros con otitis externa, utilizando los antibióticos más usados en la clínica diaria para tratar esta enfermedad. Se obtuvieron 148 muestras de secreciones óticas provenientes de 104 perros con otitis externas que fueron atendidos en una clínica veterinaria de lima, Perú, en un periodo de seis meses. Se identificaron 113 aislados como Staphylococcus $\mathrm{sp}$, de las cuales, 105 fueron CoPS. Las cepas presentaron la mayor resistencia a la ciprofloxacina (27.4\%) y la mayor sensibilidad a la nitrofurantoína $(82.1 \%)$.
\end{abstract}

Palabras clave: Staphylococcus, resistencia antimicrobiana, meticilina, otitis externa

\section{Abstract}

The coagulase-positive staphylococci (CoPS) group includes the species $S$. aureus, $S$. pseudintermedius, $S$. intermedius and S. schleiferi subsp. coagulans. The purpose of this study was to evaluate the antimicrobial resistance of CoPS isolated from dogs with external otitis, using the most commonly antibiotics prescribed in the daily clinic to treat this disease. Samples of ear secretions $(n=148)$ were obtained from 104 dogs with external otitis treated in a veterinary clinic in Lima, Peru in a period of six months. Among them, 113 isolates were identified as Staphylococcus sp, of which 105 were CoPS. The isolates showed the greatest resistance to resistance to ciprofloxacin $(27.4 \%)$ and the greatest sensitivity to nitrofurantoin $(82.1 \%)$.

Key words: Staphylococcus, antimicrobial resistance, methicillin, external otitis

\footnotetext{
${ }^{1}$ Laboratorio de Microbiología y Parasitología Veterinaria, Facultad de Medicina Veterinaria, Universidad Nacional Mayor de San Marcos, Lima, Perú

${ }^{2}$ E-mail: joel.palomino2@unmsm.edu.pe
}

Recibido: 11 de octubre de 2019

Aceptado para publicación: 28 de enero de 2020

Publicado: 31 de marzo de 2020 


\section{INTRODUCCIÓN}

La microbiota ótica de los perros se compone de bacterias, parásitos y hongos, la cual puede afectarse como consecuencia de una reacción alérgica, presencia de cuerpos extraños o neoplasias (Korbelik et al., 2019). Así mismo, los tratamientos empíricos sin haber identificado al agente infeccioso causante de la otitis externa causan una mayor alteración de la microbiota ótica, lo cual permite la sobrepoblación de bacterias oportunistas o el ingreso de bacterias ambientales (Wiebe, 2015).

Los gérmenes de mayor frecuencia en el ambiente ótico son los Staphylococcus sp. Uno de los factores de virulencia de estas bacterias es la enzima coagulasa o estafilocoagulasa, que convierte el fibrinógeno en fibrina, generando una capa de protección frente a los macrófagos (Hermans et al., 2010). Dentro del grupo de Staphylococcus coagulasa positiva (CoPS) se encuentra $S$. aureus, bacteria de importancia en la salud pública por su resistencia a los antimicrobianos (Sasaki et al., 2010). Entre otros CoPS que afectan a los animales se puede mencionar a $S$. pseudintermedius, $S$. intermedius y $S$. schleiferi subsp. coagu-lans, los cuales también han sido reportados como resistentes a la meticilina (Devriese et al., 2005; Kawakami et al., 2010; Penna et al., 2010).

Cada año se reportan más casos de bacterias multirresistentes a los antimicrobianos. El S. aureus resistente a la meticilina (SARM) se encuentra dentro del grupo de bacterias de alerta mundial, por ser el agente bacteriano de mayor importancia en las infecciones nosocomiales en medicina humana (Sakoulas et al., 2001; Merlino et al., 2002). Adicionalmente, otras especies de Staphylococcus como S. pseudintermedius y otros CoPS han mostrado ser resistentes a la meticilina (Frank et al., 2009; Weese y van Duijkeren, 2010). Además, se ha demostra- do que existe una relación de bacterias resistentes a la meticilina con la presencia del gen $m e c A$, lo cual le provee a la bacteria no solo resistencia contra los $\beta$-lactámicos, sino, además, a otros grupos de antibióticos (Bemis et al., 2006; Bannoehr et al., 2007; Schwarz et al., 2018). Sin embargo, en el Perú no se han realizado estas evaluaciones, de allí que el objetivo del presente estudio fue determinar el perfil de resistencia antimicrobiana de los aislados causantes de otitis externa en perros.

\section{Materiales y Métodos}

\section{Especímenes}

Se tomaron 148 muestras de secreción ótica a 104 perros con otitis externa, sin distinción de raza, edad ni sexo, que acudieron a la Clínica de Animales Menores de la Facultad de Medicina Veterinaria de la Universidad Nacional Mayor de San Marcos (Lima, Perú) entre enero y junio de 2018. Como criterio de exclusión para la toma de muestra fue perros que recibieron tratamiento antibiótico durante los tres días previos al muestreo. Las muestras fueron tomadas mediante hisopados de las secreciones óticas. De los 104 perros, 44 cursaron con una otitis bilateral y sus muestras fueron evaluadas de manera independiente debido a las diferencias clínicas reportadas por el médico veterinario tratante.

\section{Identificación de Staphylococcus sp}

Los hisopados de las muestras de otitis externa fueron sembrados en agar tripticasa de soya (TSA) y agar MacConkey (MC) e incubados a $37^{\circ} \mathrm{C}$ por 24 horas. Adicionalmente, se investigó la posible presencia de levaduras en los hisopados. La identificación de las bacterias del género Staphylococcus se realizó mediante características macroscópicas, tinción Gram, catalasa y oxidasa (Schleifer y Bell, 2015). 
Cuadro 1. Perfil de susceptibilidad antimicrobiana (\%) de 105 cepas de Staphylococcus coagulasa positiva (CoPS) aislados de muestras de otitis externa en perros

\begin{tabular}{lccccccccc}
\hline Interpretación & GN & OX & CL & DA & FD & CIP & N & DO & AMC \\
\hline Sensible & 76.1 & 74.4 & 74.4 & 54.7 & 82.1 & 51.3 & 59.8 & 74.4 & 74.4 \\
Intermedio & 4.3 & 0 & 0 & 16.2 & 4.3 & 11.1 & 7.7 & 5.1 & 0 \\
Resistente & 9.4 & 15.4 & 15.4 & 18.0 & 3.4 & 27.4 & 22.2 & 10.3 & 15.4 \\
\hline
\end{tabular}

GN: gentamicina, OX: oxacilina, CL: cefalexina, DA: clindamicina, FD: nitrofurantoína, CIP: ciprofloxacina, N: neomicina, DO: doxiciclina, AMC: amoxicilina con ácido clavulánico

Técnica de Kirby-Bauer e interpretado con los estándares del CLSI (2017)

\section{Staphylococcus Coagulasa Positiva}

Se realizó la prueba de coagulasa en tubo mezclando los aislados de Staphylococcus sp con plasma de conejo (Bactident ${ }^{\circledR}$ Coagulase) e incubando a $37^{\circ} \mathrm{C}$ por 4 horas. La formación de un coágulo fue considerada como positivo. Las cepas consideradas como negativas fueron dejadas a temperatura ambiente $\left(20-25^{\circ} \mathrm{C}\right)$ hasta 24 horas para confirmar el resultado como negativo (Zdove et al., 2004).

\section{Susceptibilidad Antimicrobiana}

La prueba de sensibilidad antimicrobiana se hizo siguiendo el método de difusión en disco Kirby-Bauer. Se hizo una dilución bacteriana semejante a la escala de turbidez de 0.5 de McFarland y la muestra fue sembrada en el agar Müller-Hinton con la ayuda de un hisopo estéril (Fariña et al., 2013). Se seleccionaron los antibióticos más usados en la clínica de animales de compañía para el tratamiento de otitis externa. Así, se emplearon los discos de sensibilidad antimicrobiana (Lab Oxoid) de gentamicina $(30 \mu \mathrm{g})$, cefalexina $(30 \mu \mathrm{g})$, clindamicina $(2 \mu \mathrm{g})$, nitrofurantoína $(300 \mu \mathrm{g})$, ciprofloxacina $(5 \mu \mathrm{g})$, neomicina $(30 \mu \mathrm{g})$, doxiciclina $(30 \mu \mathrm{g}) \mathrm{y}$ amoxicilina con ácido clavulánico $(20 \mu \mathrm{g} / 10$ $\mu \mathrm{g})$. Adicionalmente, se evaluó la suscepti- bilidad a la meticilina usando el disco de oxacilina (1 $\mu \mathrm{g})$ (Bemis et al., 2006). La interpretación de la susceptibilidad se categorizó de acuerdo con las tablas del Instituto de Estándares Clínicos y Laboratoriales (CLSI) (Weinstein et al., 2018).

\section{Resultados}

Se aislaron 113 (76.4\%) cepas del género Staphylococcus sp de las 148 muestras colectadas. Las cepas fueron identificadas por ser colonias blanquecinas, circulares y de bordes definidos, cocos Gram positivas agrupados en racimos, catalasa positiva y negativa a la oxidasa. Además, entre las bacterias aisladas se encontraron Pseudo-monas sp (20.3\%), Proteus sp (12.8\%), Escherichia coli (3.4\%) y Streptococcu|s sp (2.0\%). También se observó un crecimiento excesivo de Malassezia pachydermatis $(51.4 \%, 76 / 148)$.

De las 113 cepas de Staphylococcus sp, 105 fueron positivas a la prueba de coagulasa en tubo. De estas, 72.4\% (76/105) fueron positivas en las primeras 4 horas y $27.6 \%(29 / 105)$ fueron inicialmente negativas, pero resultaron positivas en el transcurso de las 24 horas. 
Los resultados a la susceptibilidad antimicrobiana demostraron que el antibiótico con mayor número de cepas resistentes fue la ciprofloxacina $(27.4 \%, 32 / 105)$, mientras que la nitrofurantoína fue el antibiótico con el menor número de cepas resistentes (3.4\%. 4/105). Por otro lado, la susceptibilidad de la oxacilina fue de $15.4 \%$ (18/105) para las cepas de Staphylococcus coagulasa positiva (Cuadro 1).

\section{Discusión}

El estudio concuerda con las investigaciones de otros autores donde Staphylococcus sp es la principal bacteria aislada en otitis externa de perros (Yamashita et al., 2005; Muñoz et al., 2012; Zur et al., 2016). No obstante, Bugden (2013) la reportó como la segunda bacteria más común en este tipo de patología.

Las pruebas bioquímicas para la identificación de especies de Staphylococcus sp no logran ser altamente específicas debido a la gran variabilidad de esta bacteria (Schleifer y Bell, 2015). Para lograr la adecuada identificación bacteriana se requiere realizar pruebas moleculares tales como la secuenciación del ARNr 16S (Rodicio y Mendoza, 2004).

Los Staphylococcus poseen genes de resistencia hacia las fluoroquinolonas (Schwarz et al., 2018). Esto, en combinación con el uso excesivo de este grupo de antibióticos para las otitis externas explicaría la elevada resistencia que muestra frente a la ciprofloxacina (Kunder et al., 2015).

La resistencia a los $\beta$-lactámicos (penicilinas y cefalosporinas) encontrados en este estudio (15.4\%) fueron similares a los reportados por Morris et al. (2006). Estos resultados fueron corroborados con la resistencia a la oxacilina, la cual se relaciona con la presencia del gen mecA (Bemis et al., 2006).
La resistencia hacia los aminoglucósidos, como la gentamicina $(9.4 \%)$ y la neomicina (22.2\%), fueron inferiores al estudio de Penna et al. (2010), quienes obtuvieron $67.1 \%$ de resistencia a la gentamicina y $87.8 \%$ a la neomicina. Debido a sus características ototóxicas, dichos antibióticos deberían ser reservados para casos de bacterias multirresistentes.

La nitrofurantoína, otro antibiótico reservado para bacterias multiresistentes, fue el antibiótico con mayor sensibilidad en el presente estudio $(82.1 \%)$. Sin embargo, se debe considerar que en otras localidades, como es el caso de Brasil donde se ha encontrado hasta $42 \%$ de resistencia a este antibiótico (Penna et al., 2010). Esto supone una terapia primaria diferente en otros países, por lo cual genera un perfil de resistencia diferente.

En algunos casos, la M. pachydermatis puede permanecer como un factor perenne y desencadenar una infección ótica secundaria (Bajwa, 2019). En este estudio se observó un sobrecrecimiento de este microorganismo en el 51.4\% (76/148) de los casos, pero debido a que no se tiene un registro citológico previo a la otitis, se hace imposible determinar si el agente desencadenante fue fúngico o bacteriano.

\section{Conclusiones}

- Las bacterias Staphylococcus sp coagulasa positivo (CoPS) fueron los principales microorganismos aislados de otitis externas caninas.

- La mayor resistencia antimicrobiana de las CoPS fue hacia la ciprofloxacina (27.4\%)

\section{Literatura Citada}

1. Bajwa J. 2019. Canine otitis externa Treatment and complications. Can Vet J. 60: 97-99. 
2. Bannoehr J, Ben Zakour NL, Waller AS, Guardabassi L, Thoday KL, van den Broek AHM, Fitzgerald JR. 2007. Population genetic structure of the Staphylococcus intermedius group: insights into agr diversification and the emergence of methicillin-resistant strains. J Bacteriol 189: 8685-8692. doi: 10.1128/JB.01150-07

3. Bemis DA, Jones RD, Hiatt LE, Ofori ED, Rohrbach BW, Frank LA, Kania $S A$. 2006. Comparison of tests to detect oxacillin resistance in Staphylococcus intermedius, Staphylococcus schleiferi, and Staphylococcus aureus isola-tes from canine hosts. J Clin Micro-biol 44: 33743376. doi: 10.1128/JCM.01336-06

4. Bugden DL. 2013. Identification and antibiotic susceptibility of bacterial isolates from dogs with otitis externa in Australia. Aust Vet J 91: 43-46. doi: 10.1111/avj.12007

5. Devriese LA, Vancanneyt M, Baele M, Vaneechoutte $M$, De Graef $E$, Snauwaert C, Cleenwerck I, et al. 2005. Staphylococcus pseudinterme-dius sp. nov., a coagulase positive species from animals. Int J Syst Evol Microbiol 55: 15691573. doi: 10.1099/ijs.0.63413-0

6. Fariña N, Carpinelli L, Samudio M, Guillén R, Laspina F, Sanabria R, Abente $S$, et al. 2013. Staphylococcus coagulasa-negativa clínicamente significativos. Especies más frecuentes y factores de virulencia. Rev Chil Infectol 30: 480-488. doi: 10.4067/S0716-10182013000500003

7. Frank LA, Kania SA, Kirzeder EM, Eberlein LC, Bemis DA. 2009. Risk of colonization or gene transfer to owners of dogs with meticillinresistant Staphylococcus pseudintermedius. Vet Dermatol 20: 496-501. doi: 10.1111/j.1365-3164.2009.00826.x

8. Hermans K, Devriese LA, Haesebrouck F. 2010. Staphylococcus. In: Pathoge-nesis of bacterial infections in animals. $4^{\text {th }}$ ed. USA: Blackwell Publishing. p. $75-89$.
9. Kawakami T, Shibata S, Murayama N, Nagata M, Nishifuji K, Iwasaki T, Fukata T. 2010. Antimicrobial susceptibility and methicillin resistance in Staphylococcus pseudintermedius and Staphylococcus schleiferi subsp. coagulans isolated from dogs with pyoderma in Japan. J Vet Med Sci 72: 1615-1619. doi: 10.1292/ jvms.10-0172

10. Korbelik J, Singh A, Rousseau J, Weese JS. 2019. Characterization of the otic bacterial microbiota in dogs with otitis externa compared to healthy individuals. Vet Dermatol 30: 228-e70. doi: 10.1111/ vde. 12734

11. Kunder DA, Cain CL, O'Shea K, Cole $S D$, Rankin SC. 2015. Genotypic relatedness and antimicrobial resistance of Staphylococcus schleiferi in clinical samples from dogs in different geographic regions of the United States. Vet Dermatol 26: 406-411. doi: 10.1111/ vde. 12254

12. Merlino J, Watson J, Barbara $R$, Beard-Pegler M, Gottlieb T, Bradbury $R$, Harbour C. 2002. Detection and expression of methicillin/oxacillin resistance in multidrug-resistant and nonmultidrug-resistant Staphylococcus aureus in Central Sydney, Australia. J Antimicrob Chemoth 49: 793-801. doi: 10.1093/jac/dkf021

13. Morris DO, Rookt KA, Shofer FS, Rankin SC. 2006. Screening of Staphy-lococcus aureus, Staphylococcus intermedius, and Staphylococcus schleiferi isolates obtained from small companion animals for antimicro-bial resistance: A retrospective review of 749 isolates (2003-04). Vet Dermatol 17: 332-337. doi: 10.1111/j.13653164.2006.-00536.x

14. Muñoz L, Molina M, Heresmann M, Abusleme F, Ulloa M, Borie C, San Martin B, Silva V, et al. 2012. Primer reporte de aislamiento de Staphylococcus schleiferi subespecie coagulans en perros con pioderma y otitis externa en Chile. Arch Med Vet 44: 261-265. doi: 10.4067/S0301-732X2012000300008 
15. Penna B, Varges $R$, Medeiros L, Martins GM, Martins RR, Lilenbaum W. 2010. Species distribution and antimicrobial susceptibility of staphylococci isolated from canine otitis externa. Vet Dermatol 21: 292-296. doi: 10.1111/j.1365-3164.2009.00842.x

16. Rodicio M, Mendoza M. 2004. Identificación bacteriana mediante secuenciación del ARNr 16S: fundamento, metodología y aplicaciones en microbiología clínica. Enferm Infec MicrCl 22: 238-245. doi: 10.1016/S0213-005X(04)73073-6

17. Sakoulas G, Gold HS, Venkataraman L, Degirolami PC, Eliopoulos GM, Qian Q. 2001. Methicillin-resistant Staphylococcus aureus: comparison of suscepti-bility testing methods and analysis of mecA-positive susceptible strains. J Clin Microbiol 39: 3946-3951. doi: 10.1128/JCM.39.11.3946-3951.2001

18. Sasaki T, Tsubakishita S, Tanaka Y, Sakusabe A, Ohtsuka M, Hirotaki S, Kawakami T, Fukata T, Hiramatsu K. 2010. Multiplex-PCR method for species identification of coagulasepositive staphylococci. J Clin Microbiol 48: 765-769. doi: 10.1128/JCM.01232-09

19. Schleifer K-H, Bell JA. 2015. Staphylococcus. In: Bergey's manual of systematics of archaea and bacteria. Springer. p. 1-43.

20. Schwarz S, Feßler AT, Loncaric I, Wu C, Kadlec K, Wang Y, Shen J. 2018. Antimicrobial resistance among Staphylococci of animal origin. Microbiol Spectr 6(4). doi: $10.1128 / \mathrm{micro-}$ biolspec.ARBA-0010-2017
21. Weese JS, van Duijkeren E. 2010. Methicillin-resistant Staphylococcus aureus and Staphylococcus pseudintermedius in veterinary medicine. Vet Microbiol 140: 418-429. doi: 10.1016/ j.vetmic.2009.01.039

22. Weinstein MP, Patel JB, Bobenchik AM, Campeau S, Cullen SK, Galas MF, Gold H, Humphries RM, Kirn TH. 2018. Performance standards for antimicrobial disk susceptibility tests. $13^{\text {th }}$ ed. Pensilvania, USA: Clinical and Laboratory Standards Institute. $92 \mathrm{p}$.

23. Wiebe VJ. 2015. Drug therapy for infectious diseases of the dog and cat. Iowa: Wiley-Blackwell. $328 \mathrm{p}$.

24. Yamashita K, Shimizu A, Kawano J, Uchida E, Haruna A, Igimi S. 2005. Isolation and characterization of staphylococci from external auditory meatus of dogs with or without otitis externa with special reference to Staphylococcus schleiferi subsp. coagulans isolates. J Vet Med Sci 67: 263-268. doi: 10.1292/jvms.67.263

25. Zdovc I, Ocepek M, Pirš T, Krt B, Pinter L. 2004. Microbiological features of Staphylococcus schleiferi subsp. coagulans, isolated from dogs and possible misidentification with other canine coagulase-positive staphylococci. J Vet Med Ser B 51: 449-454.

26. Zur G, Gurevich B, Elad D. 2016. Prior antimicrobial use as a risk factor for resistance in selected Staphylococcus pseudintermedius isolates from the skin and ears of dogs. Vet Dermatol 27: 468e125. doi: $10.1111 /$ vde. 12382 